\title{
Abundancia de perros en situación de calle y su relación con factores ambientales en Río Cuarto (Córdoba, Argentina)
}

\author{
de la Reta, M.; Muratore, M.; Perna, S.; Polop, J.; Provensal, M.C. \\ Grupo de investigación en Ecología de Poblaciones, Universidad Nacional \\ de Rio Cuarto, Córdoba, Argentina. E-mail: marinadelareta@gmail.com
}

\begin{abstract}
Resumen
de la Reta, M.; Muratore, M.; Perna, S.; Polop, J.; Provensal, M.C.: Abundancia de perros en situación de calle y su relación con factores ambientales en Río Cuarto (Córdoba, Argentina). Rev. vet. 29: 2, 113-118, 2018. El presente estudio propuso estimar la población canina callejera de Río Cuarto y establecer relaciones con factores ambientales que expliquen la distribución de los individuos. Se realizó un muestreo zonificando la ciudad $\mathrm{y}$, a través de transectas, se contabilizaron los perros en situación de calle y se registraron variables referidas a características de los individuos y del ambiente. Se estimó la abundancia poblacional y los datos obtenidos fueron comparados entre sector y sexo a través de ANOVA y test de Tukey. Para establecer la relación entre la abundancia y las características ambientales, se aplicaron análisis de agrupamientos y modelos lineales generalizados. La población estimada fue de 5.558 perros y las diferencias de dicha abundancia entre sectores y sexos fueron significativas estadísticamente. Además, los resultados sugieren que no existe una dinámica poblacional propia, y que las variables ambientales tendrían importancia en la distribución de los individuos, por lo que alterar el acceso a determinados recursos podría impactar en la población.
\end{abstract}

Palabras clave: perros callejeros, demografía, distribución espacial, ambiente.

\begin{abstract}
de la Reta, M.; Muratore, M.; Perna, S.; Polop, J.; Provensal, M.C.: Abundance of stray dogs and its relationship with environmental factors in Río Cuarto (Córdoba, Argentina). Rev. vet. 29: 2, 113-118, 2018. The present study was performed to estimate the canine population of Rio Cuarto city, and to establish possible relationships with environmental factors that may explain the distribution of individuals. Sampling was performed by dividing the city in different areas and, using transects, dogs were counted. Variables related to characteristics of individuals and the environment were recorded as well. Population was estimated and data were compared between geographic area and sex through ANOVA and Tukey's test. To establish the relationship between abundance and environmental characteristics, cluster analyzes and generalized linear models were used. The estimated population was 5,558 dogs, differences in population between sectors and sex were statistically significant. The results suggest that there is no specific population dynamics, and that environmental variables would be important in the distribution of individuals, so modifications in the access of animals to certain resources could impact in the population.
\end{abstract}

Key words: stray dogs, demography, spatial distribution, environment variables.

\section{INTRODUCCIÓN}

En general, la población de perros en situación de calle es la manifestación de una serie de factores socioeconómicos y culturales que tiene su origen en la insuficiente educación ciudadana y en una deficiente legislación sobre el impacto de los canes en el medio ambiente y en la salud pública ${ }^{3,8}$.

Se ha comprobado que el entorno urbano contiene numerosas áreas donde los perros callejeros encuen-

Recibido: 19 junio 2018 / Aceptado: 2 agosto 2018 tran protección ante diversas condiciones, hallando espacios de refugio temporal, situación que se observa en forma repetitiva en la mayoría de las ciudades de Latinoamérica ${ }^{5,12}$.

Por otro lado, la disponibilidad de alimentos para los perros es probablemente uno de los factores más importantes que influyen en la densidad poblacional, siendo la basura desechada por los seres humanos una de las principales fuentes de alimento ${ }^{3,8}$.

Desde el punto de vista sanitario, la convivencia del hombre con el perro trae riesgos para la población humana, tanto en la transmisión como en la disemi- 
nación de enfermedades zoonóticas, registrando altas tasas de incidencia y causando significativa morbilidad y mortalidad ${ }^{1}$.

Considerando tales aspectos, hoy muchas naciones están atravesando la problemática del crecimiento descontrolado de la población canina, sin embargo existen pocos estudios poblacionales y la mayoria de ellos son enfocados a determinar el número de perros con propietarios, no encontrándose datos confiables de individuos en situación de calle.

Asimismo, se considera que existen razones básicas para estimar la abundancia de la población de perros callejeros en una ciudad. En primer lugar, para evaluar la necesidad de un control poblacional, en segundo lugar, para planificar una intervención de control si fuera necesaria, y finalmente para evaluar una intervención ya realizada.

Según la Organización Mundial de la Salud (OMS) ${ }^{14}$, el valor recomendado de la relación hombre/ perro es de 10:1; sin embargo al revisar valores estudiados, se encuentra que en América Latina la Organización Panamericana de la Salud (OPS) estimó en el año 2003 una relación hombre/perro de 7,7:1 ${ }^{15}$.

Por otro lado, en Argentina, hasta la creación del Programa nacional de tenencia responsable y sanidad de perros y gatos ${ }^{17}$, los métodos empleados para controlar la población canina habían resultado descoordinados e ineficientes, además de carecer de fundamentos éticos y de un verdadero diagnóstico de situación que permita llevar a cabo un plan eficiente.

El municipio de Río Cuarto, Provincia de Córdoba, promulgó la creación de un Centro de Esterilización Municipal en el ámbito del Ente Descentralizado de Control Municipal (EDECOM), contando además con un centro de reinserción de canes en situación de maltrato y abandono, y otras ordenanzas referidas al control de perros.

Tales decisiones le valieron haber sido declarada "ciudad no eutanásica" (Ordenanza 530/09) ${ }^{6}$. A pesar de ello, estas iniciativas no se han visto sustentadas en un conocimiento real de la población canina, que permita conocer los valores de abundancia en la ciudad, cómo se distribuyen, y si la intervención que se está realizando resulta tan efectiva como presume ser, o bien debería modificarse.

Por lo expuesto, los objetivos del presente estudio fueron estimar la población total de perros callejeros en áreas públicas de la ciudad de Río Cuarto y establecer relaciones con factores ambientales. Se procedió también a caracterizar la población canina considerando estructura demográfica y caracteristicas de los individuos.

\section{MATERIAL Y MÉTODOS}

El estudio se realizó en Río Cuarto, ciudad situada al sur de la provincia de Córdoba $\left(33^{\circ} 07^{\prime} 23^{\prime \prime} \mathrm{S}\right.$ $\left.64^{\circ} 20^{\prime} 52^{\prime \prime} \mathrm{O}\right)$ y cabecera del departamento homónimo, cubriendo una superficie de $64,25 \mathrm{~km}^{2}$ y contando con 246.393 habitantes, según el Censo Nacional de Población, Hogares y Viviendas ${ }^{10}$.

Para realizar el muestreo de la población de perros, se tuvo en cuenta la definición de la Organización Mundial de Sanidad Animal (OIE) ${ }^{13}$, que distingue "perro con propietario" a aquel canino del que una persona se hace responsable; y "perro vagabundo" a todo aquél que no esté bajo control directo de una persona o al que no se le impida deambular libremente.

En el presente trabajo se utilizó una modificación en la terminología de la OIE, empleándose el término de perros en situación de calle. Con ello se designa a individuos errantes con propietario pero libres de vigilancia o restricción directa en un momento dado, así como a animales callejeros sin propietario.

Se llevó a cabo una zonificación de la ciudad para diferenciar los sectores a muestrear, teniendo en cuenta el Plan Urbano de Río Cuarto ${ }^{7}$, que considera cuatro sectores: norte, este, centro y oeste (Figura 1).

El muestreo se realizó considerando cada sector como estrato, en donde se llevaron a cabo conteos y observaciones directas de los perros a través de transectas. Cada transecta consistió en un total de 16 cuadras, y su forma fue determinada siguiendo el método de trayectos de líneas adyacentes que recomienda la Sociedad Mundial para la Protección Animal ${ }^{22}$.

La asignación de las calles que las comprendieron fue sistematizada, siendo distribuidas a lo largo y ancho de cada sector, manteniendo una distancia mínima de dos cuadras entre cada una de ellas. El orden de los sectores a muestrear fue elegido al azar.

De la misma manera se procedió con la elección del orden de las transectas ubicadas dentro de cada sector. Así, se llevó a cabo el muestreo con un total de 36
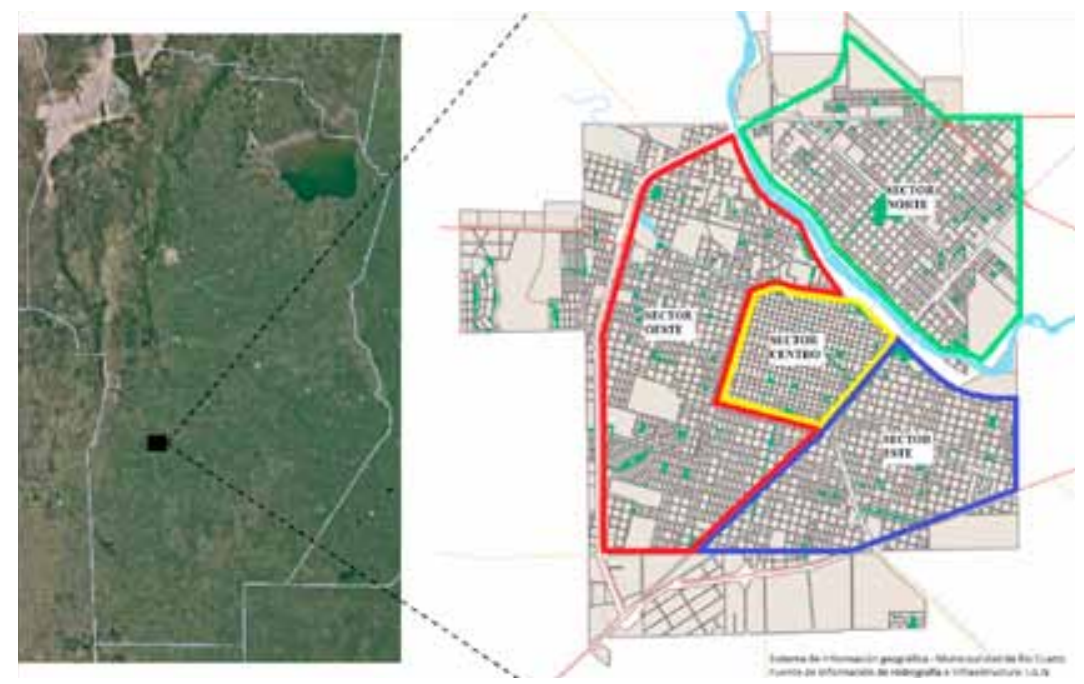

Figura 1. Zonificación de la ciudad de Río Cuarto para llevar a cabo el muestreo. 
transectas, correspondiendo 8 de ellas al sector Norte, 7 al sector Centro, 7 al sector Este, y las 14 restantes al sector Oeste.

Para el conteo de los perros se siguió la metodología propuesta por WSPA (2007) ${ }^{21}$, realizando los recorridos a pie y utilizando un "cuenta-ganado" manual. Se efectuaron los conteos de aquellos animales que se encontraron en propiedad pública al momento de la observación, cubriendo por completo la calle y sin crear disturbios que pudieran llegar a modificar el comportamiento de los individuos.

La observación se llevó a cabo durante la mañana entre las 9:00 y las 12:00 horas. Cada transecta fue recorrida en un promedio de 35 minutos y la totalidad del muestreo se realizó en un período comprendido entre abril y mayo de 2015 .

Complementariamente se registraron variables tanto referidas a características de los individuos como al ambiente. Se registró el sexo de cada individuo y su condición reproductiva. En el caso de las hembras, se determinó si estaban inactivas reproductivamente, preñadas o lactantes; y en lo machos se observó si los mismos estaban castrados o enteros.

Se determinó la edad del animal considerando dos estados: menores o mayores a un año; y se registró la raza, observando si eran animales puros o mestizos. Se registró también su aspecto sanitario, distinguiendo entre estado bueno si no exhibía lesión alguna; regular si presentaba cojera o algunas pocas lesiones en la piel $\mathrm{u}$ ojos; o malo cuando se observaba caquexia, importantes lesiones epiteliales u oculares, tumores evidentes, fracturas o patas amputadas.

Por otro lado se registró el temperamento del animal, de acuerdo a si el individuo presentaba o no alguna reacción. De esta manera se determinó si el perro presentaba reacción de susto reflejando miedo hacia el observador, reacción amigable si mostraba entusiasmo, o reacción agresiva si generaba alguna provocación como ladridos y propensión al ataque.

En el caso de las variables ambientales, se registraron aquellas que tuvieran alguna relación con la disponibilidad de agua, alimento o refugio, distinguiéndolas en cualitativas y cuantitativas. Las variables cualitativas fueron de doble estado, según si se encontraban o no presentes, relevándose seis: agua corriente, contenedores de basura, microbasurales, estacionamientos, terreno con vías de ferrocarril y calles pavimentadas. En cuanto a las variables cuantitativas, se registró el número de lugares con agua estancada y con expendios de alimento, como así también el número de bolsas de basura, de plazas y de descampados.

Los datos de abundancia obtenidos fueron cotejados entre sector y sexo a través de un ANOVA (análisis de la varianza bifactorial), luego de haber transformado los datos a logaritmo +1 , para que cumplieran los supuestos de normalidad y homogeneidad de la varianza. Para detectar entre cuáles sectores había diferencias significativas se aplicó a posteriori el test de Tukey.
La estimación del número total de perros en situación de calle se obtuvo multiplicando el número promedio de perros por cuadra, por la cantidad total de cuadras de la ciudad. Del mismo modo, se procedió a estimar la abundancia de canes en cada sector por separado.

Las variables consideradas para la caracterización de la población canina fueron expresadas en números y porcentajes, y la relación entre la abundancia poblacional y las distintas características ambientales se llevó a cabo mediante diferentes análisis. Una primera aproximación fue la elaboración de un análisis de agrupamiento entre las transectas, considerando las variables cuantitativas y aplicando el coeficiente de distancia euclideana y la técnica de ligamiento completo.

Por otro lado, se aplicaron modelos lineales generalizados (MLG) para analizar la relación de la abundancia de perros con las variables cuantitativas y cualitativas, así como para relacionar de a pares una variable cuantitativa con una cualitativa. Para la selección del mejor modelo, en cada caso, se utilizó el criterio de información de Akaike (AIC), una medida de la calidad relativa de un modelo estadístico, para un conjunto de datos. Todos los análisis estadísticos mencionados se realizaron utilizando el programa $R$ Project 3.2.1.

\section{RESULTADOS Y DISCUSIÓN}

Abundancia poblacional y caracterización de la población canina. Durante el muestreo se contabilizaron 821 perros en situación de calle, obteniendo un promedio total de 23 perros por transecta y 1,43 perros por cuadra, difiriendo este valor según el sector de la ciudad (Tabla 1). Las diferencias de la abundancia de perros en situación de calle entre sectores y sexos fueron significativas estadísticamente $(\mathrm{F}=7,741 ; \mathrm{gl}=3 ; \mathrm{p}=$ 0,0002 y $\mathrm{F}=7,561 ; \mathrm{gl}=1 ; \mathrm{p}=0,0077$, respectívamente), siendo no significativa la interacción sector-sexo $(\mathrm{F}=$ $0,778 ; \mathrm{gl}=3 ; \mathrm{p}=0,5105)$.

La relación hombre/perro en situación de calle fue de 44,3:1, una proporción muy pequeña comparada al valor recomendado por la OMS ${ }^{14}$ de 10:1. Sin embargo, el valor indicado es referido al número total de perros en una localidad. No existe ningún estudio comparable en donde se exprese una relación hombre/perro que sea específica de los perros en situación de calle.

Tabla 1. Abundancia de perros en situación de calle según sectores de la ciudad de Río Cuarto, por transecta y por cuadra (período Abril-Mayo 2015).

\begin{tabular}{lcccc}
\hline sector & $\begin{array}{c}\text { perros por } \\
\text { transecta }(\overline{\mathrm{x}})\end{array}$ & DE & $\begin{array}{c}\text { perros por } \\
\text { cuadra }(\overline{\mathrm{x}})\end{array}$ & $\mathrm{n}$ \\
\hline centro $(a)$ & 9,00 & $\pm 4,00$ & 0,56 & 307 \\
norte $(b c)$ & 21,75 & $\pm 8,28$ & 1,36 & 1.092 \\
oeste $(b)$ & 22,43 & $\pm 18,33$ & 1,40 & 2.247 \\
este $(c)$ & 38,57 & $\pm 23,34$ & 2,41 & 2.274 \\
total ciudad & 22,81 & $\pm 17,97$ & 1,43 & 5.558 \\
\hline
\end{tabular}

Letras en cursiva representan diferencias significativas, $\mathrm{p}<0,05$. ( $\mathrm{x}$ : media aritmética, DE: desvío estándar, n: abundancia). 
A pesar de ello, esta variable es importante ya que a medida que aumentan los perros con relación a la población humana, se incrementan los riesgos para la salud pública por el estrecho contacto que existe entre ambas poblaciones.

En cuanto a la estructura poblacional, en todos los sectores se contabilizó un mayor número de perros machos que hembras, registrando una relación macho/ hembra de 1,6:1. De los machos, se observó que solo 20 individuos estaban castrados, sobre 454 enteros. Con respecto a las hembras, se registraron 289 perras inactivas reproductivamente, 8 lactantes y 1 preñada.

Analizado la estructura etaria, de los 821 individuos relevados con edad determinada, solo el 3\% resultó ser menor a un año de edad. Por lo anterior, se evidencia que los datos registrados con relación a la estructura y proporción de sexo no representan una dinámica poblacional propia.

El muestreo se llevó a cabo en los meses de abril y mayo, época no propicia para encontrar cachorros, pero sí juveniles o hembras recientemente preñadas. Si se adjudica que el total de los individuos registrados como ejemplares menores a 1 año proviene de apareamientos de los perros en situación de calle, habría que suponerlos provenientes del estro primaveral. Pero de esta manera, ese $3 \%$ de juveniles y el bajo número de hembras preñadas registradas no estarían representando lo esperado en función a la proporción de perros adultos encontrados.

Es evidente que podría haberse producido una alta mortalidad entre las crías nacidas en esa oportunidad, dada la susceptibilidad de los cachorros a algunas enfermedades infecciosas, o bien pensarse que una porción significativa de cachorros fue recogida y convertida en perros domiciliarios. Esto se complementaría con un traspaso de perros adultos hacia la calle. Ese movimiento entre el hogar que recoge un gran porcentaje de las crías nacidas en la calle y posteriormente las devuelve como adultos podría explicar los valores de abundancia registrados.
Asimismo, el dato observado sobre el porcentaje de machos no esterilizados, a pesar de los esfuerzos del municipio en términos de castración gratuita y de una numerosa difusión de las campañas, sumado a que la población callejera podría provenir mayormente de los individuos que se abandonan y no de la reproducción in situ, induce a repensar esta medida como única técnica de control en la ciudad.

En cuanto al aspecto sanitario, el 95,7\% de los canes presentó un aspecto bueno, el 2,9\% aspecto regular y el 1,6\% un aspecto malo. Se sabe, sin embargo, que la mayoría de los perros callejeros no reciben tratamientos veterinarios, y su permanencia en la calle representa un potencial factor de riesgo para la salud de las personas, al actuar como reservorios de enfermedades y como fuente de contaminación ambiental ${ }^{3-5,9}$. Debe tenerse en cuenta que en Argentina se han realizado estudios en caninos, resultando positivos ante agentes como Leptospira, Toxocara, Ancylostoma, Trichuris, Dipylidium, Taenia ${ }^{18,19}$ e incluso Ancylostoma en la propia ciudad en estudio ${ }^{20}$.

Por otro lado, la mayoría de los animales no presentó reacción alguna, siendo muy pocos los registros de perros asustados, amigables o agresivos. Específicamente, solo el 6\% de los individuos presentaron un carácter agresivo. Dicho dato puede contrastarse con los antecedentes de mordeduras denunciadas en el año 2014 en la ciudad, donde solo el 32\% de ellas fueron ocasionadas por perros sin dueño (comunicación personal, 2015). La información sobre ataques caninos varía según los estudios por diferentes causas y se considera que la descripción del contexto constituye uno de los apartados menos analizados o estudiados y que debería mejorarse o profundizarse a fin de reforzar las pautas preventivas $^{2,3,16}$.

Relación entre abundancia poblacional y variables ambientales. El dendrograma obtenido del análisis de agrupamientos se muestra en la Figura 2. En el mismo pueden diferenciarse 4 grupos principales con-

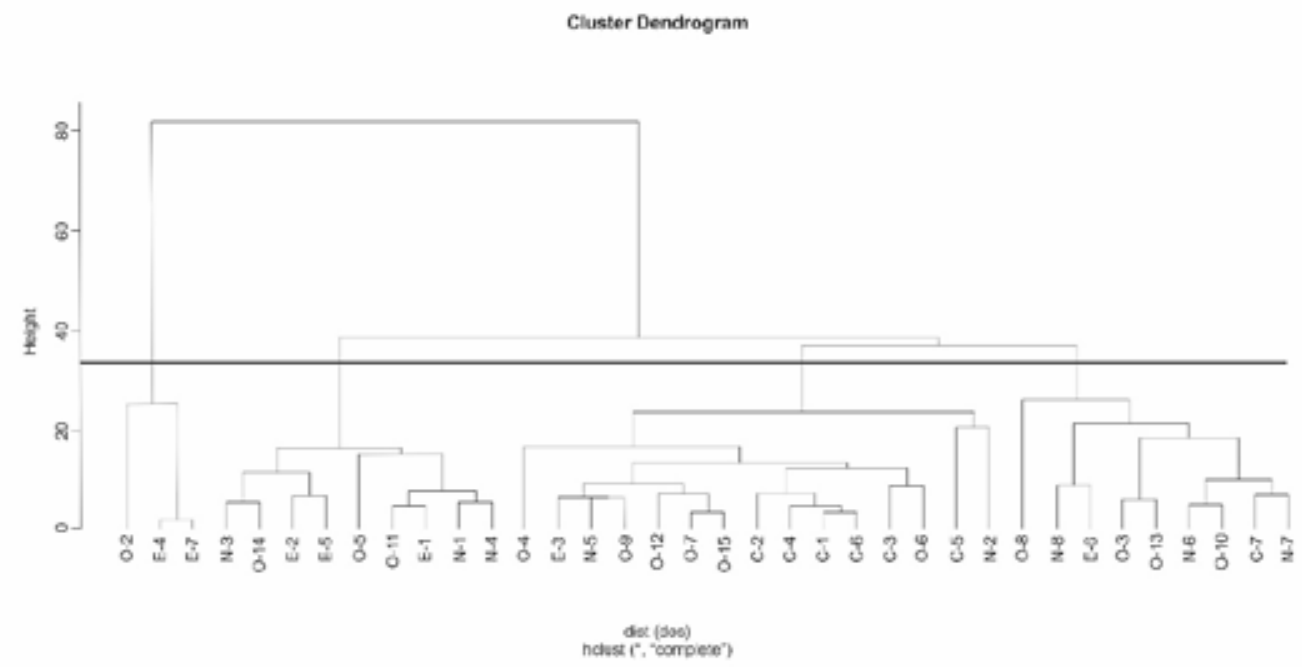

Figura 2. Dendrograma obtenido del análisis de agrupamiento entre las transectas, considerando variables ambientales cuantitativas. 
formados por transectas que pertenecían a diferentes sectores. Así, se pudo observar que el primer grupo contiene las transectas que se correspondieron con los valores más elevados de abundancia de animales (entre 70 y 81 individuos).

El segundo grupo comprende las transectas representadas por valores de abundancia entre 24 y 39 individuos. Las del tercer grupo presentaron un número de perros que varió entre 4 y 23. El último grupo incluyó aquellas transectas donde se registraron entre 6 y 26 perros.

De este análisis surge que las variables ambientales consideradas, y su relación entre ellas, podrían tener más importancia en la determinación de la abundancia poblacional que la mera diferenciación sectorial de la ciudad, a pesar de que dicha abundancia haya sido significativamente diferente entre sectores. Posiblemente, si se sectorizase la ciudad de otra manera y teniendo en cuenta variables socioeconómicas, culturales y de urbanización del lugar, podrían obtenerse datos más contundentes en relación con las diferencias halladas.

Al relacionar las variables cuantitativas respecto a la abundancia de perros en situación de calle, se encontró que el número de bolsas de basura y de descampados está relacionada positivamente y el número de lugares con agua estancada y de plazas presenta una relación inversa; no evidenciándose relación significativa con el número de lugares de expendios de alimentos (Tabla 2).

Por otro lado, de los MLG obtenidos, al considerar las variables cualitativas, se seleccionó el Modelo 1 por presentar el menor AIC (criterio de información de Akaike). Sin bien éste incluye todas las variables, la abundancia de perros se relacionó positivamente con la presencia de microbasurales y negativamente con la presencia de pavimento y contenedores, no existiendo significación con el agua corriente, estacionamientos o terrenos con vías de ferrocarril (Tabla 3).

Relacionando de a pares las variables cuantitativas y cualitativas con el número de perros, se detectó que a medida que aumenta la cantidad de bolsas de basura la abundancia de perros en situación de calle fue mayor; sin embargo, ante la presencia de contenedores en esos lugares la abundancia fue menor. Asimismo, el mayor número de canes en relación al número de bolsas de basura, también disminuyó cuando las calles son pavimentadas.

En cuanto a la presencia de plazas como refugio temporal para los perros, se encontró que éstas se asocian a bajos números de animales. Además, cuando se registra la existencia de contenedores de basura en dichas plazas, la abundancia fue menor aún. Por otro lado, se observó que el número de descampados presentaba una relación positiva con la abundancia poblacional y
Tabla 2. Valores del modelo lineal generalizado donde se relaciona la abundancia de perros con variables ambientales cuantitativas.

\begin{tabular}{lrrrc}
\hline variable & estim. & DE & valor $\mathrm{z}$ & \multicolumn{1}{c}{$\mathrm{p}(<\mathrm{z})$} \\
\hline agua estancada & $-0,11$ & 0,02 & $-4,50$ & $6,78 \mathrm{e}^{-6 *}$ \\
expendio alimento & 0,01 & 0,01 & 0,66 & 0,51 \\
bolsa basura & 0,04 & 0,01 & 5,95 & $2,65 \mathrm{e}^{-9 *}$ \\
plaza & $-0,21$ & 0,03 & $-6,48$ & $8,98 \mathrm{e}^{-1 *}$ \\
descampado & 0,02 & 0,01 & 3,90 & $9,69 \mathrm{e}^{-5 *}$ \\
\hline
\end{tabular}

estim: estimativo. DE: desvío estándar. Desvianza residual $=-0,3362$. Los asteriscos indican diferencias significativas. El "e" es el exponencial (p.ej.: 6,78 e $\mathrm{e}^{-6}=0.000000678$ ). El signo negativo de los estimadores significa que la relación es inversa, o sea (p.ej.) si hay agua estancada entonces la probabilidad de encontrar perros (o la abundancia de perros) es menor.
Tabla 3. Valor $\mathrm{p}$ de los modelos lineales generalizados y los respectivos valores de AIC, donde se relaciona la abundancia de perros con variables ambientales.

\begin{tabular}{lccccccc}
\hline \multirow{2}{*}{ modelo } & \multicolumn{5}{c}{ valor $\mathrm{p}(<\mathrm{z})$} \\
\cline { 2 - 6 } & $\mathrm{ac}$ & $\mathrm{c}$ & $\mathrm{m}$ & $\mathrm{es}$ & $\mathrm{f}$ & $\mathrm{p}$ & \multirow{2}{*}{ AIC } \\
\hline todos & 0,94 & $1,23 \mathrm{e}^{-5 *}$ & $0,61 \mathrm{e}^{-3 *}$ & 0,88 & 0,18 & $6,00 \mathrm{e}^{-6 *}$ & 490,37 \\
ac & $0,027 *$ & & & & & & 564,56 \\
$\mathrm{e}$ & & & & 0,35 & & & 568,78 \\
$\mathrm{c}$ & & $5,26 \mathrm{e}^{-8 *}$ & & & & & 538,22 \\
$\mathrm{f}$ & & & & & 0,229 & & 568,20 \\
$\mathrm{ac}+\mathrm{m}$ & 0,73 & & $5,76 \mathrm{e}^{-11 *}$ & & & 516,16 \\
$\mathrm{~m}+\mathrm{p}$ & & & $1,04 \mathrm{e}^{-6 *}$ & & 0,20 & 495,75 \\
\hline
\end{tabular}

ac: agua corriente; c: contenedor; m: microbasural; es: estacionamiento; $\mathrm{f}$ : terreno con vías de ferrocarril; p: pavimento.AIC (criterio de información de Akaike $)=470,75$. Los asteriscos indican diferencias significativas. Exponentes: similar significado al expuesto en Tabla 2.

el número de perros era todavía mayor ante la presencia de microbasurales en dichos descampados.

No sucedió lo mismo cuando hubo contenedores cerca de los descampados, ya que se observó una menor abundancia. Los resultados obtenidos indican que las bolsas de basura podrían estar ofreciendo suministros e incentivando la presencia de perros en los lugares en los que ella se registra. Estos datos coinciden con lo observado en otras ciudades ${ }^{8}$, donde la cantidad de perros fue significativamente menor en lugares donde la basura se encontraba en contenedores, restringiendo de esa manera el acceso a la basura.

Es probable que el comportamiento humano relacionado a las variables mencionadas sea la causa mayor detrás de la explicación de la abundancia de la población de perros en situación de calle. Otro factor por considerar podría ser la frecuencia de recolección de basura domiciliaria, que no resulta igual en todos los sectores de la ciudad.

Por otro lado, los resultados relacionados a las variables de refugio sugieren que los perros se encuentran en mayor abundancia en lugares de menor urbanización, viéndose potenciados por la presencia de basura y 
microbasurales. Alterar el acceso a los recursos podría generar un impacto en la población de perros en situación de calle al desincentivar la vagancia oportunista, así como también podría llegar a reducir potencialmente la supervivencia de aquéllos que dependen de estos recursos.

Este estudio representaría el punto inicial de aquellos pasos necesarios para poder conocer las causas de la distribución y la abundancia de la población de perros en situación de calle de la ciudad de Río Cuarto, dado que la técnica de muestreo empleada se mostró robusta para el registro confiable de los datos reportados.

De esta manera, tanto la metodología desarrollada como los resultados obtenidos fueron transferidos a la Dirección General de Bromatología, Medio Ambiente y Zoonosis, perteneciente al EDECOM, como informe técnico a fin de que la entidad posea una metodología simple y estandarizada para recoger información que pueda ser útil y confiable para la toma de decisiones.

Agradecimientos. Al personal del Ente Descentralizado de Control Municipal (EDECOM), especialmente a Paulo Rizo por la participación en los muestreos y a la Mgter. Susana Ferrero, por su colaboración.

\section{REFERENCIAS}

1. Acha P, Szyfres B. 2001. Zoonosis y enfermedades transmisibles comunes al hombre y los animales. Publicación cientifica OPS/OMS 580, 3ra ed., Washington, p.398.

2. Alfieri A, Marro A, Seguesso A, Schiaffino L, Bin LE, Pirles ME. 2007. Caracterización de mordeduras de perros en Arequito. Anales Jornadas de Divulgación Técnico-Cientificas (Fac.Cs.Veterinarias, Casilda), p. 13-14.

3. Álvarez E, Domínguez J. 2001. Programa para el control integral de la población canina. Revista AMMVEPE (México) 12: 83-91.

4. Beck A. 1973. The ecology of stray dogs: A study of free-ranging urban animals. On line:http://docs.lib.purdue.edu/press_ebooks/?utm_source=docs.lib.purdue. edu $\% 2$ Fpress_ebooks\%2F4\&utm_medium $=$ PDF\&utm campaign $=$ PDFCoverPages.

5. Bögel K. 1987. Guidelines for dog rabies control. Publ. World Health Organization, Geneva. www.who.int/iris/ handle/10665/61215

6. Concejo Deliberante de la Ciudad de Rio Cuarto. 2009. Ordenanza 530. Río Cuarto, Córdoba, Argentina.

7. Concejo Deliberante de la Ciudad de Rio Cuarto. 2011. Ordenanza 1.082. Río Cuarto, Córdoba, Argentina.

8. Ibarra L, Espínola F, Echeverría M. 2006. Factores relacionados con la presencia de perros en las calles de la Ciudad de Santiago, Chile. Avances en Ciencias Veterinarias 21: $21-26$.
9. Ibarra L, Espínola F, Echeverría M. 2006. Una prospección a la población de perros existente en las calles de la Ciudad de Santiago, Chile. Avances en Ciencias Veterinarias 21: 33-39.

10. INDEC (Instituto Nacional de Estadística y Censos). 2010. Censo Nacional de Población, Hogares y Viviendas 2010. Buenos Aires, Argentina.

11. Kitala P, McDermott J, Kyule M, Gathuma J. 2000. Community-based active surveillance for rabies in Machakos District, Kenya. Prev Vet Med 44: 73-85.

12. Molina JL, Faigenbaum A, Castro JR, Gastrel H, Ruggia R. 2006. Estimación de la población de caninos vagabundos en la ciudad de Buenos Aires, octubre-noviembre 2006. Publ. Instit. Zoonosis Luis Pasteur, Minist. Salud, Buenos Aires.

13. OIE, Organización Mundial de Sanidad Animal. 2010. El control de las poblaciones de perros vagabundos. En: Código Sanitario para los Animales Terrestres. www.oie. int/doc/ged/D12823.PDF

14. OMS, Organización Mundial de la Salud y WSPA, World Society for the Protection of Animals. 1990. Guiderlines for dog population management. Geneva. www.who.int/iris/handle/10665/61417

15. OPS, Organización Panamericana de la Salud. 2003. Resumen de la situación de los programas de rabia en la América Latina. Informe final. RIMSA 13/INF/2, p. 36-70. bvs1.panaftosa.org.br/local/file/textoc/IX_Redipra_esp. pdf

16. Palacio J, León M, García BS. 2005. Aspectos epidemiológicos de las mordeduras caninas. Gac Sanit (España) 19: 50-58.

17. Presidencia de la Nación. 2011. Decreto 1.088. Programa nacional de tenencia responsable y sanidad de perros y gatos, Buenos Aires. www.anima.org.ar

18. Rubel D, Wisnivesky C. 2010. Contaminacion fecal canina en plazas y veredas de Buenos Aires, 1991-2006. MEDICINA (Buenos Aires) 70: 355-363

19. Tealdo M, Romero G, Autrey C, Samartino L. 2007. Serología positiva a Leptospira interrogans, serovar cynopteri en caninos de la ciudad de Buenos Aires, Argentina. In Vet 9: 59-65.

20. Tolosa PJ, Chiaretta A, Lovera H. 2006. El parasitismo. Una asociación interespecifica, Ed.Universidad Nacional Río Cuarto, 138 p. ISBN 9506653933.

21. WSPA, Sociedad mundial para la protección animal. 2007. Censando poblaciones de perros deambulantes: guía metodológica. 21 p. xa.yimg.com/.../resources Companion\%2520Animals_true_Surveying-roaming-dog

22. WSPA, Sociedad mundial para la protección animal. 2007. 24 p.www.icam-coalition.org/.../guia_para_el_manejo_humanitario_de_poblaciones_caninas. 